\title{
Investigating Auditory Spectral and Temporal Resolution Deficits in Children with Reading Difficulties
}

DOI: $10.3766 /$ jaaa. 17142

Kiri Mealings*

Sharon Cameron*

\begin{abstract}
Background: The types of reading difficulties experienced by children are highly heterogeneous in nature, which makes diagnosis and intervention difficult. Over the past 30 years, there has been much debate over the cause of dyslexia. The two most popular theories for phonological deficits in dyslexia are the rate-processing constraint hypothesis, which relates to short timescale processing, and the temporal sampling framework hypothesis, which relates to longer timescale processing.

Purpose: To investigate the relationship between sublexical (i.e., nonword) reading skills and auditory spectral and temporal resolution patterns in children with reading difficulties using the Phoneme Identification Test (PIT) and the Parsing Syllable Envelopes Test (ParSE). These tests were developed to assess the rate-processing constraint and the temporal sampling framework hypotheses, respectively. We hypothesized that a proportion of children who have sublexical reading difficulties may have an underlying auditory-resolution deficit which may impact their ability to form letter-sound correspondences. We predicted that children's sublexical reading difficulties may not be explained by one theory, but instead that both theories may describe different types of reading difficulties found in different children. We also hypothesized that children with lexical (i.e., irregular word) reading difficulties but intact sublexical reading skills would not show atypical results on PIT or ParSE.
\end{abstract}

Research Design: Behavioral experimental clinical study with children who have reading difficulties.

Study Sample: Sixteen children with nonword, irregular word, or mixed reading difficulties diagnosed by the Castles and Coltheart Test 2.

Data Collection and Analysis: Children completed a test battery consisting of a hearing screen and tests of reading, auditory resolution, phonological awareness, attention, spatial auditory processing, auditory memory, and intelligence. Categorization and correlational analyses were conducted.

Results: All four children with a pure sublexical reading deficit also had an auditory-resolution deficit. Four of seven children with a mixed reading deficit had an auditory-resolution deficit. Only one of five children with a lexical reading deficit had an auditory-resolution deficit. Individual children's specific deficits were related to either rate processing $(n=5)$ or temporal sampling $(n=4)$, but never both. Children's nonword reading scores were strongly correlated with their performance on the PIT in noise, but not with the PIT in quiet or the ParSE. Children's irregular word scores were not significantly correlated with their performance on the PIT in quiet or in noise, or the ParSE, as hypothesized. Strong correlations were also found between children's nonword scores and their phonological awareness scores.

Conclusions: The results of this study suggest that neither the rate-processing hypothesis nor the temporal sampling framework is the single cause of reading difficulties in children. Instead, both of these hypotheses are likely to account for different types of reading deficits found in children. This is an important finding as the specific mechanisms driving different reading impairments must be identified to create tools to better diagnose and treat different types of reading difficulties. Further investigation of the PIT and ParSE as potential diagnostic tools for specific auditory-resolution-based reading difficulties in a larger group of children is currently underway.

*National Acoustic Laboratories, Sydney, Australia

Corresponding author: Kiri Mealings, National Acoustic Laboratories, Australian Hearing Hub, Macquarie University, Macquarie Park, NSW 2109, Australia; Email: kiri.mealings@nal.gov.au

This research is funded by the Australian Government through the Department of Health. 
Key Words: amplitude modulation, rate processing, reading difficulties, spectro-temporal processing, temporal sampling

\begin{abstract}
Abbreviations: $\mathrm{CC} 2$ = Castles and Coltheart Test 2; CV = consonant-vowel speech segment; GPC = grapheme-phoneme conversion; NMF = number memory forward; NMR = number memory reversed; ParSE = Parsing Syllable Envelopes Test; PIT = Phoneme Identification Test; PITN = Phoneme Identification Test (noise condition); PITQ = Phoneme Identification Test (quiet condition); UR = uncertainty region (measure on the PIT)
\end{abstract}

\section{INTRODUCTION}

$\mathrm{C}$ hildren experiencing reading difficulties in the classroom are a heterogeneous group, making diagnosis and intervention difficult (McArthur et al, 2013). The most commonly accepted model about learning to reading aloud is the dual-route cascade model (Coltheart et al, 2001). In English, there are two types of words that children need to learn to read (McArthur et al, 2013). The first type of words is regular words. These words can be sounded out using grapheme-phoneme conversion (GPC) rules (e.g., "bed"). The second type of words is irregular words. These words do not follow GPC rules and need to be learned by sight (e.g., "yacht"). The dual-route cascade model describes the two ways that children learn English words. The sublexical route involves children learning the GPC rules so that they can decode regular words. The lexical route involves children memorizing whole words by sight and adding them to their mental lexicon (Coltheart, 2005; McArthur et al, 2013). To become a skilled reader, children need to become sufficient in using both routes simultaneously with equal strength (Coltheart, 2005). That is, children need to be able to use the sublexical route to sound out new words and then transfer them to the lexicon so they can be read quickly by sight along with the irregular words using the lexical route.

Developmental dyslexia refers to a disorder whereby children have difficulty learning to read despite having normal intelligence, no neurological abnormalities, and adequate reading instruction (Hulme and Snowling, 2009; McArthur et al, 2013). Dyslexia can be categorized into three main types: phonological reading difficulties, surface reading difficulties, and mixed reading difficulties (Castles and Coltheart, 1993; McArthur et al, 2013). Children with a phonological reading difficulty have poor sublexical reading ability but normal lexical reading ability. That is, they can use the lexical reading route to memorize whole words, but have difficulty using GPC rules to sound out words. By contrast, children with a surface reading difficulty can use GPC rules to sound out words, but have difficulty using the lexical route to memorize irregular words. Most children, however, have a mixed reading difficulty, which means that they have trouble reading both regular words using the sublexical route and irregular words using the lexical route (McArthur et al, 2013; Birch, 2016).

Deficits in phonological processing are largely thought to underlie poor reading performance in most people with phonological reading difficulties (Hämäläinen et al, 2012). A recent meta-analysis of phonological skills and their role in learning to read found that phonemic awareness had the strongest correlation with word reading ability (Melby-Lervåg et al, 2012). However, there is much debate over what timescale these phonological deficits are related to (Hämäläinen et al, 2012; Halliday et al, 2017). Currently, the two most popular theories for phonological deficits in dyslexia are the rate-processing constraint hypothesis, which relates to short timescale processing (Tallal, 1980; 1984; 2004; see also Farmer and Klein, 1995, for a review), and the temporal sampling framework hypothesis (Goswami, 2011), which relates to longer timescale processing.

The rate-processing constraint hypothesis proposes that a subset of children with dyslexia have a deficit in processing brief or rapid auditory information, such as sounds that occur over tens of milliseconds. This type of processing is important for fine-grained acoustic analysis such as rapid formant transitions that are essential for identifying different stop phonemes, for example, /b/, /d/, and /g/ (Tallal and Gaab, 2006; Chang et al, 2010). Gamma neural oscillations predominantly in the left auditory cortex are believed to correspond to phonemic-level processing (Poeppel, 2003; Giraud and Poeppel, 2012). A rate-processing deficit is thought to lead to inaccurate phoneme perception and, therefore, result in the development of less precise phonological representations of these phonemes (see Halliday et al, 2017; Hämäläinen et al, 2012, for a review).

Alternatively, the temporal sampling framework hypothesis (Goswami, 2011) proposes that children with dyslexia have a deficit in detecting slower modulation rates, such as sounds that occur over hundreds of milliseconds. This type of processing is important for understanding speech prosody. The acquisition of phonological awareness follows a hierarchical model (Anthony et al, 2003). Children's awareness of syllables is usually present when they are three to four years old, whereas phoneme awareness only develops once a child is taught to read and write (see Ziegler and Goswami, 2005, for a 
review). A temporal sampling deficit is thought to cause problems with the segmentation of speech into smaller elements, such as syllables (see Halliday et al, 2017; Hämäläinen et al, 2012 for a review). Theta neural oscillations predominantly in the right auditory cortex are believed to moderate syllable-level processing (Poeppel, 2003; Giraud and Poeppel, 2012). According to the temporal sampling framework hypothesis, atypical temporal integration of syllables in the right hemisphere may form the auditory basis of the phonological and language deficits experienced by people with dyslexia (Goswami, 2011). As phonemic awareness only begins once children learn to read, the temporal sampling framework hypothesis anticipates that phonemic deficits in children with dyslexia are a consequence of poor reading rather than a core feature (Ziegler and Goswami, 2005; Cutini et al, 2016).

Several studies have been conducted to assess these two theories, and have largely yielded mixed results (see Protopapas, 2013; Ramus and Ahissar, 2012, for reviews). Numerous studies have been conducted by Tallal et al that support the rate-processing hypothesis, and many studies have been conducted by Goswami et al supporting the temporal sampling hypothesis (see Farmer and Klein, 1995; Ramus and Ahissar, 2012; Protopapas, 2013, for reviews). There have also been various studies outside of these laboratories that give results in support of one or the other hypotheses, for example, Vandermosten et al (2011), Serniclaes et al (2004), Poelmans et al (2012), Cutini et al (2016), and Lorenzi et al (2000).

A recent review by Protopapas (2013) on the rateprocessing and temporal sampling theories found many studies that did not fit with either the rate-processing hypothesis or the temporal sampling framework hypothesis. This review included studies that found no difference between dyslexics and controls on rapid processing and/or temporal sampling, and studies that showed differences between dyslexics and controls on psychoacoustic tests that were outside of both of these theories. In addition, Protopapas (2013) highlighted issues with several studies that did match one of the theories but that had unreliable results due to poor experimental design and poor control in the studies.

Children's speech-perception-in-noise abilities have also been found to be associated with their reading ability. Hornickel et al (2009) found that children with poor phonological awareness and poor speech-in-noise perception have reduced subcortical differentiation of [ba], [da], and [ga] stop consonant stimuli relative to good performers. White-Schwoch et al (2015) found that preschool children's neural coding of consonants in noise predicts their future reading skills. Ziegler et al (2009) found that children's speech perception deficits only manifest in noise, not in quiet. This brought them to the conclusion that the core deficit of dyslexics is be- cause of a lack of speech robustness in the presence of noise.

In light of these mixed findings and the highly heterogeneous nature of reading difficulties, we believe that dyslexia may not be able to be explained by one theory, and hence that there may not be a single cause for all reading difficulties. Both the rate-processing constraint hypothesis and the temporal sampling framework hypothesis are examples of single distal cause models as they propose that their respective auditoryprocessing deficit is the sole cause of dyslexia (Halliday et al, 2017). As a result, they are seen to be competing theories, so evidence for one is interpreted as evidence against the other (Protopapas, 2013). However, it may be that both theories describe different types of dyslexia that are found in different people. Therefore, a multifactor model that takes into account multiple sensory, cognitive-linguistic, and higher order cognitive processes is required (Boets et al, 2007). Our aim is to gain a better understanding of the more specific mechanisms driving different impairments so we can create more sensitive methods to better diagnose and treat different types of reading difficulties.

For the purpose of this study, we posit that a proportion of children with sublexical reading difficulties have an underlying auditory-resolution deficit arising from the difficulties with the central auditory nervous system decoding and reassembling the rapidly changing frequency and amplitude components of speech, as occurring for children with cochlear hearing loss (Kyle and Harris, 2010). Consequently, continued exposure to low-resolution speech sounds may result in indistinct speech sound templates being reinforced in the cortical areas responsible for speech perception and recognition. Such deficits in children may lead to difficulties in mapping speech sounds to orthographic symbols using GPC rules. Without these clear auditory templates, we believe that deficits in phonetically based reading skills will result.

Cameron et al recently developed two new tests to assess fine spectro-temporal processing and temporal envelope cues in children with suspected auditoryprocessing deficits (Cameron et al, 2018a,b). The Phoneme Identification Test (PIT) (Cameron et al, 2018a) assesses how well a child can perceptually categorize speech sounds based on their rapidly changing formant frequencies (i.e., it tests the rate-processing constraint hypothesis). The Parsing Syllable Envelopes Test (ParSE) (Cameron et al, 2018b) assesses how well a child can perceive where syllables begin and end based on the amplitude modulations at the boundaries (i.e., it tests the temporal sampling framework hypothesis). Therefore, the aim of this study was to use these tests to investigate the relationship between sublexical reading skills and auditory-resolution patterns in children 
with reading difficulties, with the ultimate aim to see if these tests have potential to be used as diagnostic tools for specific types of reading difficulties. It was hypothesized as follows:

- A proportion of children who demonstrate impaired sublexical route reading (i.e., phonological reading difficulties or mixed reading difficulties) would also have an auditory-resolution deficit, either in the fast-rate processing of formant frequency changes (measured by the PIT) or in the temporal sampling of slower amplitude modulations (measured by the ParSE). Furthermore, if a child showed a deficit on the PIT, we expected that it would be greater when the stimuli were presented in noise than in quiet (Hornickel et al, 2009; Ziegler et al, 2009; Cameron et al, 2018a).

- The children who demonstrated impaired lexical route reading but intact sublexical route reading (i.e., surface reading difficulties) would not show an auditory-resolution deficit on the PIT or ParSE.

- Children's performance on the PIT and ParSE would be positively correlated with their sublexical reading skills (with the correlation being stronger when the PIT is presented in noise), but would not be correlated with their lexical reading skills. In addition, we expected that children's sublexical reading skills would be correlated with their phonological awareness skills (Melby-Lervåg et al, 2012).

\section{METHOD}

pproval for the study was granted from the AusA tralian Hearing Human Research Ethics Committee and the New South Wales Department of Education.

\section{Participants}

A total of 44 children were recruited from three Sydney primary schools for this research. These children were nominated by their teachers as children with reading difficulties. Note that for the purpose of our study, we will use the term "children with reading difficulties" to describe these children as they did not necessarily have a formal diagnosis of dyslexia. Of these 44 children, 16 children qualified to be included in the study. Children whose parents reported that they had an attention, language, or learning problem on the study consent form were excluded from participating $(n=15)$. Eighteen children began the testing but were excluded because of either failing the hearing screening $(\mathrm{n}=2)$, passing the reading test $(n=14)$, failing the spatial auditory-processing test $(\mathrm{n}=1)$, or having a suspected attention deficit $(n=1)$. The demographics of the 16 children included in the study were as follows: males $=$ 10 , females $=6$; age range $=8$ years 2 months to 11 years 4 months, mean age $=$ aged 9 years 7 months. All children were monolingual English speakers.

\section{Test Battery}

The tests outlined in the following paragraphs were used for the study. Testing was completed over two sessions on separate days, each lasting up to an hour. Children were given breaks throughout the testing when needed, and received stickers after they completed each test for encouragement and motivation. All testing was completed in a quiet room at the child's school one-toone with the researcher.

\section{Hearing Screening}

Children's hearing was screened on the day of testing, and only those who passed the pure tone audiometric screening test progressed on to the test battery. All children included in the study had normal hearing defined as equal to, or better than, $20 \mathrm{~dB}$ HL at all octave frequencies from 500 to $8000 \mathrm{~Hz}$ using an Interacoustics Audio Traveller A222 portable audiometer (Middelfart, Denmark) with Telephonics TDH 39P audiometric headphones (Huntington, NY) in H7A Peltor cups (3M, St. Paul, MN).

\section{Reading Test}

The Castles and Coltheart Test 2 (CC2) (Castles et al, 2009) was used to assess what type of reading deficit the children had. The CC2 assesses children's ability to read regular words that can be sounded out (e.g., "bed"), nonwords that can be sounded out (e.g., "gop"), and irregular words that cannot be sounded out and need to be learned by sight (e.g., "yacht"). The children were assessed on the three types of words, but we were only concerned about their performance on the nonwords and irregular words for this study, as these are what categorize a child as having a phonological, mixed, or surface reading difficulty. A child was considered to have a reading difficulty if they scored less than average results, which is defined by the CC2 as a $z$ score of $<-1$ on any of the subtests.

\section{Auditory-Resolution Tests}

\section{PIT}

The PIT (Cameron et al, 2018a) was used to assess children's ability to process rapid formant transitions. The child heard synthesized /ba/ or /da/ syllables over Sennheiser HD215 circumaural headphones (Hanover, Germany) and was asked to select on a 
Microsoft Surface Pro 3 touchscreen computer (Microsoft, China) which sound he/she heard using a twoalternative forced-choice procedure. The /ba/ and /da/ CV syllables differed only in the second formant frequency along an 11 step-continuum (i.e., between $0 \%$ and $100 \%$-representing an ideal $/ \mathrm{ba} /$ and $/ \mathrm{da} /$, respectively). The children completed the test in both a quiet condition (PIT quiet condition; PITQ) and a noise condition at a $0 \mathrm{~dB}$ signal-to-noise ratio (PIT noise condition; PITN). The software automatically fitted psychometric functions to individual data. Performance was determined by the width of the uncertainty region (UR), which is the width between the points at which the psychometric function has a value of $0.12-0.88$. This width represents the continuum where responses were neither clearly /ba/ nor /da/. Shallower psychometric function slopes reflected greater uncertainty. The $z$ score for the UR was calculated by the software. A child was considered to have an auditory-resolution deficit if the $z$ score was $<-1.67$ (equivalent to a percentile rank and standard score of 5). For more information on PIT, including analyses of normative and test-retest reliability data, see Cameron et al (2018a).

\section{ParSE}

The ParSE (Cameron et al, 2018b) was used to assess children's ability to recognize syllable boundaries using a self-masking amplitude modulation detection paradigm. The child heard, through Sennheiser HD215 circumaural headphones, a steady-state /a/ vowel which was modulated into two or three pseudosyllables using notches with modulation depths varying between $0 \%$ and $100 \%$ along an 11-step continuum. The child was asked to select on a Microsoft Surface Pro 3 touchscreen computer (Microsoft) whether they heard one, two, or three syllables using an adaptive three-alternative forced-choice procedure. The software automatically fitted psychometric functions to individual data. A child's performance was calculated using the upper boundary of the uncertainty region, which is the modulation depth at which syllables can be detected with $88 \%$ accuracy. Shallower psychometric function slopes reflected greater uncertainty. A child was considered to have an auditory-resolution deficit if the $z$ score was $<-1.67$. For more information on the ParSE, including analyses of normative and test-retest reliability data, see Cameron et al (2018b).

\section{Phonological Awareness Test}

The Comprehensive Test of Phonological ProcessingSecond Edition Alternate Phonological Awareness Subtest (Wagner et al, 2013) was used to assess the children's ability to put together (i.e., blend) nonwords from individual speech sounds, and also to divide (i.e., segment) whole nonwords into their individual speech sounds. The test stimuli was prerecorded by a female Australian-English speaker (the second author) and played to the child out loud at a comfortable level using a Microsoft Surface Pro 3 touchscreen computer (Microsoft). Percentiles were calculated for the blending nonwords and segmenting nonwords subtests individually.

\section{Attention Test}

The Developmental NEuroPSYchological AssessmentSecond Edition Inhibition Subtest (Korkman et al, 2007) was used as a screener to exclude children from the study who had possible attention deficits. The test was administered in accordance to the instructions using two iPads (one for the researcher and one for the child). The software calculated scaled scores for naming, inhibition, and switching tasks taking into account both the child's accuracy and their completion time. Children needed to get scaled scores of greater than five on each measure (which is equivalent to a $z$ score $>-1.67)$ to be included in the study.

\section{Spatial Auditory-Processing Test}

The Listening in Spatialized Noise-Sentences Test (Cameron and Dillon, 2009) measures the ability of people to use the interaural time and levels spatial cues that help differentiate a target talker from distracting talkers. The test is used to diagnose children with spatial processing disorder (Cameron and Dillon, 2007a,b; 2008). For this study, we only used the high cue condition to screen out children who may have a spatial processing disorder or greater-than-normal listeningin-noise difficulties. The test was administered over Sennheiser HD215 circumaural headphones using a Microsoft Surface Pro 3 touchscreen computer (Microsoft). The child was asked to repeat the sentences spoken by the target speaker which sounded like it was coming directly from in front of them while there were distracting talkers which sounded like they were standing on either side of them. Children were required to get a $z$ score $>-1.67$ to continue in our study.

\section{Auditory Memory Test}

The Test of Auditory Processing Skills-Third Edition Number Memory Forward (NMF) and Number Memory Reversed (NMR) (Martin and Brownell, 2005) subtests were used to assess children's short-term memory and working memory, respectively. The test stimuli was prerecorded by a female AustralianEnglish speaker (the second author) and played to the children at a comfortable level over the inbuilt speaker of a Microsoft Surface Pro 3 touchscreen computer (Microsoft). The children heard strings of numbers and were asked to 
recall them in the same order that they were presented (NMF), and in the reverse order that they were presented (NMR). Percentiles and scaled scores were calculated for the NMF and NMR subtests individually.

\section{Intelligence Test}

The Wechsler Abbreviated Scale of IntelligenceSecond Edition Vocabulary and Matrix Reasoning Subtests (Weschler, 2011) was used as a screener to exclude children from the study who had lower than average intelligence. The test was administered in accordance with the published testing protocol. The two subtest scores were calculated manually and combined to give an overall intelligence percentile. Children needed to achieve a percentile rank greater than five (which is equivalent to a $z$ score $>-1.67$ ) to be included in the study.

\section{RESULTS}

$\mathrm{T}$ his section reports the results of the study. All statistical analyses were performed using Statistica version 10.

\section{Reading Test}

The $z$ scores on the reading test for the participating children are shown in Table 1 . Four children had a $z$ score $<-1$ on nonwords only (i.e., a phonological reading difficulty), seven children had a $z$ score $<-1$ on both nonwords and irregular words (i.e., a mixed reading difficulty), and five children had a $z$ score $<-1$ on irregular words only (i.e., a surface reading difficulty).

\section{Auditory-Resolution Tests}

The $z$ scores for the auditory-resolution tests of participating children are also shown in Table 1. Children were considered to fail an auditory-resolution test if they had a $z$ score $<-1.67$. All four children with a phonological reading difficulty had an auditory-resolution deficit. Two failed the PITQ and PITN, one failed the PITN only, and one failed the ParSE only. Of the seven children with a mixed reading difficulty, four had an auditory-resolution deficit. Two of these children failed the PITQ and PITN, and two failed the ParSE. Of the five children with a surface reading deficit, only one child had an auditory-resolution deficit. This child failed the ParSE. Note one child (P1) in the phonological group and one child in the surface group (P16) had unreliable results outside acceptable confidence intervals on the ParSE, and as such, their results on this measure were not included in the analysis.

\section{Standardized Test Results}

The children's individual results for phonological awareness (blending nonwords and segmenting nonwords), attention (naming, inhibition, and switching), spatial auditory processing, auditory memory (NMF and NMR), and intelligence are shown in Table 2 along

Table 1. Reading and Auditory-Resolution Results for Participating Children

\begin{tabular}{|c|c|c|c|c|c|c|c|c|c|c|}
\hline \multirow[b]{2}{*}{$\begin{array}{l}\text { Reading } \\
\text { Difficulty }\end{array}$} & \multirow{2}{*}{\multicolumn{2}{|c|}{ Participant Age }} & \multicolumn{2}{|c|}{ Reading Result } & \multicolumn{3}{|c|}{ Auditory-Resolution Result } & \multicolumn{3}{|c|}{ Auditory-Resolution Deficit } \\
\hline & & & $\begin{array}{c}\text { Nonword } \\
\text { z Score }\end{array}$ & $\begin{array}{l}\text { Irregular } \\
\text { Word } \\
\text { z Score }\end{array}$ & $\begin{array}{c}\text { PITQ } \\
\text { UR } \\
\text { z Score }\end{array}$ & $\begin{array}{l}\text { PITN UR } \\
\text { z Score }\end{array}$ & $\begin{array}{l}\text { ParSE } \\
\text { UBUR } \\
\text { z Score }\end{array}$ & $\begin{array}{l}\text { Rate- } \\
\text { Processing } \\
\text { Deficit } \\
\text { (Quiet) }\end{array}$ & $\begin{array}{c}\text { Rate- } \\
\text { Processing } \\
\text { Deficit } \\
\text { (Noise) }\end{array}$ & $\begin{array}{c}\text { Temporal } \\
\text { Sampling } \\
\text { Deficit }\end{array}$ \\
\hline Phonological & P1 & $10 ; 1$ & $-1.37^{\star}$ & -0.83 & $-1.85^{\star}$ & $-2.88^{*}$ & - & $\checkmark$ & $\checkmark$ & \\
\hline & P2 & $11 ; 4$ & $-1.66^{\star}$ & -0.87 & $-2.10^{\star}$ & $-2.36^{*}$ & -0.23 & $\checkmark$ & $\checkmark$ & \\
\hline & P3 & $9 ; 4$ & $-1.17^{\star}$ & -0.20 & -1.00 & $-2.30^{\star}$ & 0.40 & & $\checkmark$ & \\
\hline & P4 & $10 ; 5$ & $-1.21^{\star}$ & -0.14 & -0.13 & 0.12 & $-1.84^{\star}$ & & & $\checkmark$ \\
\hline \multirow[t]{7}{*}{ Mixed } & P5 & $8 ; 9$ & $-1.71^{*}$ & $-1.66^{\star}$ & $-2.47^{\star}$ & $-2.54^{*}$ & 0.65 & $\checkmark$ & $\checkmark$ & \\
\hline & P6 & $10 ; 11$ & $-1.81^{\star}$ & $-1.90^{\star}$ & $-2.32^{\star}$ & $-4.02^{*}$ & -0.50 & $\checkmark$ & $\checkmark$ & \\
\hline & P7 & $9 ; 11$ & $-1.14^{*}$ & $-1.09^{*}$ & 0.74 & -0.59 & $-1.94^{*}$ & & & $\checkmark$ \\
\hline & P8 & $10 ; 8$ & $-1.14^{\star}$ & $-2.01^{\star}$ & 0.90 & -0.90 & $-1.70^{\star}$ & & & $\checkmark$ \\
\hline & P9 & $8 ; 2$ & $-1.16^{\star}$ & $-1.87^{\star}$ & 0.36 & -0.15 & 1.41 & & & \\
\hline & P10 & $10 ; 0$ & $-1.64^{\star}$ & $-1.53^{*}$ & 0.34 & 0.17 & 0.22 & & & \\
\hline & P11 & $10 ; 10$ & $-1.61^{\star}$ & $-1.56^{\star}$ & -0.30 & 0.28 & 0.50 & & & \\
\hline \multirow[t]{5}{*}{ Surface } & P12 & $8 ; 10$ & -0.29 & $-1.25^{\star}$ & -0.75 & -0.35 & $-2.76^{\star}$ & & & $\checkmark$ \\
\hline & P13 & $8 ; 5$ & -0.59 & $-1.87^{\star}$ & 0.26 & 1.37 & -0.30 & & & \\
\hline & P14 & $8 ; 6$ & -0.80 & $-1.48^{*}$ & 0.47 & -0.86 & 0.95 & & & \\
\hline & P15 & $8 ; 10$ & 0.41 & $-1.25^{\star}$ & 0.34 & 0.40 & 0.81 & & & \\
\hline & P16 & $9 ; 3$ & 0.09 & $-1.67^{\star}$ & -1.13 & 0.55 & - & & & \\
\hline
\end{tabular}


Table 2. Standardized Test Results for Participating Children

\begin{tabular}{|c|c|c|c|c|c|c|c|c|c|c|c|}
\hline \multirow{2}{*}{$\begin{array}{l}\text { Reading } \\
\text { Difficulty }\end{array}$} & \multirow[b]{2}{*}{ Participant } & \multirow[b]{2}{*}{ Age } & \multicolumn{2}{|c|}{ CTOPP-2 Percentile Rank } & \multirow{2}{*}{$\begin{array}{l}\text { LiSN-S } \\
\text { z Scores }\end{array}$} & \multicolumn{3}{|c|}{ NEPSY-II Scaled Scores } & \multicolumn{2}{|c|}{ TAPS-3 Percentile Rank } & \multirow{2}{*}{$\begin{array}{c}\text { WASI-II } \\
\text { Percentile } \\
\text { Rank }\end{array}$} \\
\hline & & & Blending & Segmenting & & Naming & Inhibition & Switching & NMF & NMR & \\
\hline \multirow[t]{4}{*}{$\overline{\text { Phonological }}$} & P1 & $10 ; 1$ & 16 & 50 & 0.20 & $6^{*}$ & 8 & 7 & 25 & 16 & $13^{*}$ \\
\hline & P2 & $11 ; 4$ & $1^{\star \star}$ & 16 & -0.87 & 15 & 12 & 10 & 25 & 37 & 55 \\
\hline & P3 & $9 ; 4$ & 84 & 75 & -0.06 & 8 & 9 & 10 & 37 & 37 & 34 \\
\hline & P4 & $10 ; 5$ & 37 & 95 & -0.20 & 8 & 10 & 12 & 84 & 84 & 87 \\
\hline \multirow[t]{7}{*}{ Mixed } & P5 & $8 ; 9$ & $9^{*}$ & 25 & $-1.39^{\star}$ & $6^{*}$ & 9 & 9 & $9^{*}$ & 25 & 45 \\
\hline & P6 & $10 ; 11$ & 50 & 91 & 0.24 & 14 & 9 & 9 & 50 & 37 & 53 \\
\hline & P7 & $9 ; 11$ & 50 & 37 & -0.78 & $6^{*}$ & 12 & 9 & $5^{*}$ & $9^{*}$ & 23 \\
\hline & P8 & $10 ; 8$ & 63 & 75 & 0.67 & 9 & 7 & 11 & 16 & 25 & 70 \\
\hline & P9 & $8 ; 2$ & 37 & 37 & -0.70 & 14 & 9 & 11 & 63 & 63 & 34 \\
\hline & P10 & $10 ; 0$ & 16 & 25 & 0.10 & 11 & 9 & 10 & 25 & 37 & 39 \\
\hline & P11 & $10 ; 10$ & 25 & 50 & -0.20 & $6^{*}$ & 7 & 10 & 16 & 16 & 25 \\
\hline \multirow[t]{9}{*}{ Surface } & P12 & $8 ; 10$ & 50 & 75 & $-1.35^{\star}$ & 12 & 12 & 9 & 37 & 50 & 73 \\
\hline & P13 & $8 ; 5$ & 75 & 75 & $-1.04^{*}$ & 7 & 8 & 10 & 50 & 16 & 55 \\
\hline & P14 & $8 ; 6$ & 37 & 84 & -0.30 & 11 & 13 & 8 & 16 & $9^{*}$ & 87 \\
\hline & P15 & $8 ; 10$ & 84 & 98 & 0.19 & 7 & 11 & 10 & 50 & 37 & 18 \\
\hline & P16 & $9 ; 3$ & 84 & 91 & 0.51 & 11 & 8 & 8 & 16 & 75 & 55 \\
\hline & & Mean & 44.88 & 62.44 & -0.31 & 9.44 & 9.56 & 9.56 & 32.75 & 35.81 & 47.88 \\
\hline & & SD & 27.53 & 27.83 & 0.64 & 3.16 & 1.90 & 1.26 & 21.77 & 22.59 & 23.21 \\
\hline & & Min & 1 & 16 & -1.39 & 6 & 7 & 7 & 5 & 9 & 13 \\
\hline & & Max & 84 & 98 & 0.67 & 15 & 13 & 12 & 84 & 84 & 87 \\
\hline
\end{tabular}

with the mean, standard deviation, minimum score, and maximum score across children on each test. Table 2 also indicates children's scores that were less than average (i.e., $z$ score $<-1$; percentile rank $\leq 15$; scaled score $<7)$ and well below average $(z$ score $<-1.67$; percentile rank $\leq 5$; scaled score $<5$ ).

\section{Correlations}

Pairwise correlations were calculated between all test scores. The correlations between the reading test and auditory-resolution tests were as follows: the children's nonword scores were significantly correlated with their performance on the PIT in noise $\left[r(16)=0.58, r^{2}=\right.$ $0.34, p=0.02]$, but were not correlated with their performance on the PIT in quiet $\left[r(16)=0.36, r^{2}=0.13, p=\right.$ $0.17]$ or on the ParSE $\left[r(14)=-0.09, r^{2}=0.01, p=\right.$ $0.75]$. The children's irregular word scores were not significantly correlated with their performance on the PIT in quiet $\left[r(16)=-0.15, r^{2}=0.02, p=0.58\right]$ or in noise $\left[r(16)=-0.16, r^{2}=0.03, p=0.55\right]$, or on the ParSE $\left[r(14)=-0.22, r^{2}=0.05, p=0.45\right]$, as hypothesized. These correlation results are shown graphically in Figures 1A-C. Strong correlations were also found between children's nonword scores and their phonological awareness scores (blending and segmenting nonwords) as expected, given that phonological awareness is an important skill for reading decoding (Melby-Lervåg et al, 2012). The results of all significant correlations between variables are shown in Table 3 .

\section{DISCUSSION}

$\mathrm{T}$ he overall aim of this study was to investigate the relationship between sublexical reading skills and spectral and temporal resolution patterns in children with reading difficulties. More specifically, we investigated the link between the rate-processing hypothesis (Tallal, 1980) and the temporal sampling framework hypothesis (Goswami, 2011) with children's reading skills using the PIT (Cameron et al, 2018a) and ParSE (Cameron et al, 2018b), respectively. The ultimate aim of this study was to assess if these tests have the potential to be used as diagnostic tools for specific types of reading difficulties.

First, it was hypothesized that a proportion of children who demonstrated impaired sublexical route reading (i.e., phonological reading difficulties or mixed reading difficulties) would also have an auditoryresolution deficit, either in the fast-rate processing of formant frequency changes (measured by the PIT) or in the temporal sampling of slower amplitude 

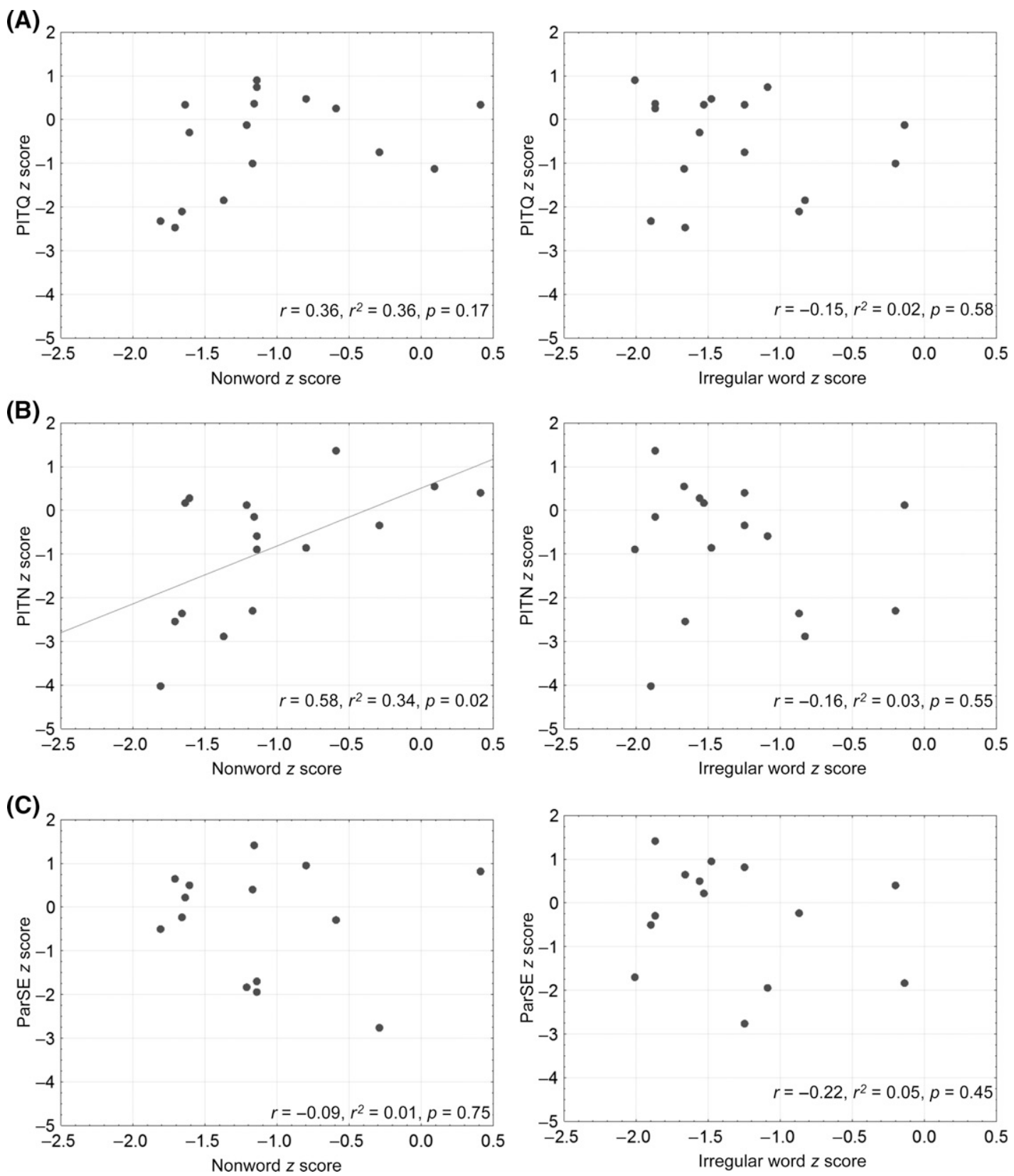

Figure 1. Correlation results between the auditory-resolution test results and nonword and irregular word reading test results for (A) PITQ, (B) PITN, and (C) ParSE.

modulations (measured by the ParSE). In line with our hypothesis, we found that all four children with pure phonological reading difficulties had an auditoryresolution deficit. We also found that four of seven children with mixed reading difficulties had an auditoryresolution deficit. Interestingly, all eight of these children with auditory-resolution difficulties had either a rate-processing deficit $(\mathrm{n}=5)$ or a temporal sampling deficit $(n=3)$, but not both. This suggests that there may be two different mechanisms at work here, each of which are related to reading difficulties but in different ways. Furthermore, for those children that did show a rate-processing deficit, this deficit was worse in noise as hypothesized, given the results of Cameron et al (2018a) and Ziegler et al (2009).

Second, it was hypothesized that the children who demonstrated impaired lexical route reading but intact sublexical route reading (i.e., surface reading difficulties) would not show an auditory-resolution deficit on the PIT or ParSE. This was because we believe that auditory-resolution deficits are related to phonological (i.e., sublexical) reading difficulties, so if the sublexical reading route is intact, then we predicted that the 
Table 3. Significant Correlation Results between Test Variables

\begin{tabular}{llllll}
\hline Variables & $\mathrm{n}$ & $r$ & $r^{2}$ & $p$ & Correlation Strength \\
\hline Nonwords vs. PITN & 16 & 0.58 & 0.34 & 0.019 & Strong \\
Nonwords vs. blending & 16 & 0.72 & 0.52 & 0.002 & Strong \\
Nonwords vs. segmenting & 16 & 0.61 & 0.37 & 0.013 & Strong \\
PITQ vs. PITN & 16 & 0.74 & 0.54 & 0.001 & Strong \\
Blending vs. segmenting & 16 & 0.74 & 0.55 & 0.001 & Strong \\
Attention switching vs. NMF & 16 & 0.57 & 0.33 & 0.020 & Strong \\
NMF vs. NMR & 16 & 0.61 & 0.37 & 0.012 & Strong \\
\hline
\end{tabular}

children should have typical auditory-resolution capabilities. This hypothesis was correct for four of the five children with surface reading difficulties. However, one child with surface reading difficulties demonstrated a temporal sampling deficit against our hypothesis. Future work is needed with a larger sample of children to investigate this further.

Third, it was hypothesized that children's results on the PIT and ParSE would be positively correlated with their sublexical (i.e., nonword) reading test results, with the correlation being stronger when the PIT was presented in noise. Conversely, it was hypothesized that children's performance on the PIT and ParSE would not be correlated with their lexical (i.e., irregular word) reading skills. As predicted, children's PIT and ParSE results were not correlated with their irregular word reading skills, suggesting that auditory-resolution deficits are not related to lexical reading. However, only children's PITN results were correlated with their nonword reading skills, not their PITQ or ParSE results. Given our finding above that children had either a rate-processing deficit or a temporal sampling deficit, trying to find correlations across all participants may have washed out some of the relationships between these variables. Instead, it would be beneficial to conduct correlations treating the two groups of children with rate-processing versus temporal sampling deficits separately. Unfortunately, we did not have a sufficient sample size to do that in the present study; however, plans are underway to investigate these relationships in a larger group of children using an expanded reading test battery. This is especially important in evaluating the ParSE as a diagnostic tool as the correlation of this test with nonword reading in the present study was only $r=-0.09$. In regard to the PITN correlating with nonword reading but not the PITQ, Ziegler et al (2009) found that children with dyslexia only exhibit clear speech perception deficits in noise but not in quiet. They concluded that the core deficit of dyslexics is likely to be due to a lack of speech robustness in the presence of noise. However, four of the five children in our study who failed the PITN also failed the PITQ. In addition, the children's PITQ results were highly correlated with their PITN results. This points more toward children having a core rate-processing deficit that is exacerbated in noise, rather than the deficit only being related to noise.

Finally, we also found that children's sublexical reading skills were correlated with their phonological awareness skills. This supports the large amount of evidence of the relationship between phonological awareness and children's reading ability (see Melby-Lervåg et al, 2012, for a review).

As this study was an initial investigation into auditoryresolution deficits and reading difficulties using two new tests, there are several limitations of the study. Although the total sample size $(n=16)$ is representative of studies in this area (see, for example, a review by Hämäläinen et al [2012]), once the children were grouped into their type of reading difficulty and then type of auditory-resolution deficit, the numbers in each group were too small to run statistical analyses for specific groups, so only observational comparisons could be made at this level. As several trends were observed, it would be worthwhile conducting a larger study to draw more definitive relationships between types of reading difficulties and types of auditory-resolution deficits. In addition, the criteria for inclusion in this study were that children were not well below average on their attention or intelligence scores (i.e., had a scaled score or percentile rank equivalent to a $z$ score $>-1.67$ ). There were a few children, however, who had below average attention (P1, P5, P7, and P11), intelligence (P1), and/or memory (P5, P7, and P14), which may have influenced their individual results even though a correlation between these scores and PIT and ParSE results was not found. Therefore, for future studies, it may be worthwhile making the inclusion criteria more strict and only including children within the average range (i.e., $-1<z$ score $<+1$ ).

Another limitation of the study was that it used a correlational analysis, so no conclusions about cause and effect could be drawn. This is a limitation of many studies as cause and effect relationships are difficult to determine (Protopapas, 2013). However, determining this relationship is vital to understanding the nature of children's reading difficulties and remediating them. Hopefully, continual developments in the field of neuroscience and neuroimaging will make it more possible to 
investigate cause and effect directional relationships in the future. As the PIT and ParSE are both auditoryprocessing tests, we think that poor performance on these tests is likely to be due to problems with the auditory pathway which results in indistinct representations. However, future functional connectivity studies are needed to assess whether the children with auditoryresolution problems on the PIT or ParSE have problems with the auditory processing of speech sounds, or problems with the access of intact speech sound representations, or potentially problems with both.

\section{CONCLUSIONS}

$\mathrm{T}$ his study provided an initial exploration of the link between the rate-processing hypothesis (Tallal, 1980) and the temporal sampling framework hypothesis (Goswami, 2011) with children's reading skills using the PIT (Cameron et al, 2018a) and ParSE (Cameron et al, 2018b), respectively. Although this correlational study cannot determine the direction of these relationships, the results suggest that neither the rate-processing hypothesis nor the temporal sampling framework is the single cause of reading difficulties in children as not all children in the study sample demonstrated either of these deficits as these hypotheses propose. Instead, these results and the general heterogeneity of reading difficulties point to different types of reading difficulties being driven by different deficits. This is in line with our thoughts that reading difficulties may not be able to be explained by one theory, and that there may not be a single cause for all reading difficulties. This is an important concept to grasp as future studies are needed to identify the specific mechanisms driving different reading impairments so we can create more sensitive tests to better diagnose and treat different types of reading difficulties. Our study provides initial evidence for both the rapid auditory-processing hypothesis and the temporal sampling framework hypothesis as separate theories describing different types of reading difficulties found in different children. This study also shows promise for the PIT and ParSE as potential diagnostic tools for specific reading difficulties related to spectro-temporal and temporal-envelope processing deficits. Further investigation of these tests in a larger group of children with reading difficulties is currently underway and includes a larger test battery of reading tests to examine other potential links between auditory-resolution deficits and more specific reading difficulties. If the PIT and ParSE continue to show promise in these larger studies and are adopted as diagnostic tools, then there is also potential for auditory training programs to be developed to remediate spectro-temporal and temporalenvelope processing deficits in children with reading difficulties.
Acknowledgments. The authors would like to thank Mark Seeto for statistical advice and also the NSW Department of Education and the primary schools that took part in this research. The participation of the children and their families is also appreciated.

\section{REFERENCES}

Anthony JL, Lonigan CJ, Driscoll K, Phillips BM, Burgess SR. (2003) Phonological sensitivity: a quasi-parallel progression of word structure units and cognitive operations. Read Res $Q$ 38(4): $470-487$.

Birch SL. (2016) Prevalence and profile of phonological and surface subgroups in college students with a history of reading disability. J Learn Disabil 49(4):339-353.

Boets B, Wouters J, van Wieringen A, Ghesquière P. (2007) Auditory processing, speech perception and phonological ability in preschool children at high-risk for dyslexia: a longitudinal study of the auditory temporal processing theory. Neuropsychologia 45(8): $1608-1620$.

Cameron S, Dillon H. (2007a) Development of the listening in spatialized noise-sentences test (LISN-S). Ear Hear 28(2):196-211.

Cameron S, Dillon H. (2007b) The listening in spatialized noisesentences test (LISN-S): test-retest reliability study. Int $J$ Audiol $46: 145-153$.

Cameron S, Dillon H. (2008) The listening in spatialized noisesentences test (LISN-S): comparison to the prototype LISN and results from children with either a suspected (central) auditory processing disorder or a confirmed language disorder. J Am Acad Audiol 19(5):377-391.

Cameron S, Dillon H. (2009) Listening in spatialized noisesentences test (LiSN-S) (version 2.4). Murten, Switzerland: Phonak Communications AG.

Cameron S, Chong-White N, Mealings KT, Beechey T, Dillon H, Young T. (2018a) The phoneme identification test (PIT) for assessment of spectral and temporal discrimination skills in children: development, normative data and test-retest reliability studies. J Am Acad Audiol 29:135-150.

Cameron S, Chong-White N, Mealings KT, Beechey T, Dillon H, Young T. (2018b) The parsing syllable envelopes (ParSE) test for assessment of amplitude modulation discrimination skills in children: development, normative data and test-retest reliability studies. J Am Acad Audiol 29:151-163.

Castles A, Coltheart M. (1993) Varieties of developmental dyslexia. Cognition 47(2):149-180.

Castles A, Coltheart M, Larsen L, Jones P, Saunders S, McArthur G. (2009) Assessing the Basic Components of Reading: A Revision of the Castles and Coltheart Test with New Norms (CC2). Sydney, Australia: Motif.

Chang EF, Rieger JW, Johnson K, Berger MS, Barbaro NM, Knight RT. (2010) Categorical speech representation in human superior temporal gyrus. Nat Neurosci 13(11):1428-1432.

Coltheart M. (2005) Modeling reading: the dual-route approach. In: Snowling MJ, Hulme C, eds. The Science of Reading: A Handbook. Oxford: Blackwell Publishing Ltd., 6-23

Coltheart M, Rastle K, Langdon R, Ziegler JC. (2001) DRC: A dual route cascaded model of visual word reecognition and reading aloud. Psychol Rev 108(1):204-256. 
Cutini S, Szűcs D, Mead N, Huss M, Goswami U. (2016) Atypical right hemisphere response to slow temporal modulations in children with developmental dyslexia. Neuroimage 143:40-49.

Farmer ME, Klein RM. (1995) The evidence for a temporal processing deficit-linked to dyslexia: a review. Psychol Bull Rev 2(4):469-493.

Giraud A-L, Poeppel D. (2012) Cortical oscillations and speech processing: emerging computational principals and operations. Nat Neurosci 15(4):511-517.

Goswami U. (2011) A temporal sampling framework for developmental dyslexia. Trends Cogn Sci 15(1):3-10.

Halliday LF, Tuomainen O, Rosen S. (2017) Auditory processing deficits are sometimes necessary and sometimes sufficient for language difficulties in children: evidence from mild to moderate sensorineural hearing loss. Cognition 166:139-151.

Hämäläinen JA, Salminen HK, Leppanen PHT. (2012) Basic auditory processing deficits in dyslexia: systematic review of the behavioral and event-related potential/field evidence. $J$ Learn Disabil 46(5):413-427.

Hornickel J, Skoe E, Nicol T, Zecker S, Kraus N. (2009) Subcortical differentiation of stop consonants relates to reading and speech-innoise perception. Proc Natl Acad Sci U S A 106(31):13022-13027.

Hulme C, Snowling M. (2009) Developmental Disorders of Language, Learning and Cognition. London: UK: Wiley-Blackwell.

Korkman M, Kirk U, Kemp S. (2007) Developmental NEuroPSYchological Assessment-Second Edition (NEPSY-II). London, UK: Pearson Clinical Assessment.

Kyle FE, Harris M. (2010) Predictors of reading development in deaf children: a 3-year longitudinal study. J Exp Child Psychol 107(3):229-243.

Lorenzi C, Dumont A, Fullgrabe C. (2000) Use of temporal envelope cues by children with developmental dyslexia. J Speech Lang Hear Res 43(6):1367-1379.

Martin N, Brownell R. (2005) Test of Auditory Processing SkillsThird Edition (TAPS-3). Novato, CA: Academic Therapy Publications.

McArthur G, Kohnen S, Larsen L, Jones K, Anandakumar T, Banales E, Castles A. (2013) Getting to grips with the heterogeneity of developmental dyslexia. Cogn Neuropsychol 30(1):1-24.

Melby-Lervåg M, Lyster S-AH, Hulme C. (2012) Phonological skills and their role in learning to read: a meta-analytic review. Psychol Bull 138(2):322-352.

Poelmans H, Luts H, Vandermosten M, Boets B, Ghesquiere P, Wouters J. (2012) Auditory steady state cortical responses indicate deviant phonemic-rate processing in adults with dyslexia. Ear Hear 33(1):134-143.

Poeppel D. (2003) The analysis of speech in different temporal integration windows: cerebral lateralization as "asymmetric sampling in time". Speech Commun 41(1):245-255.

Protopapas A. (2013) From temporal processing to developmental language disorders: mind the gap. Philos Trans $R$ Soc B Biol Sci 369(1634):1-11.

Ramus F, Ahissar M. (2012) Developmental dyslexia: the difficulties of interpreting poor performance, and the importance of normal performance. Cogn Neuropsychol 29(1-2):104-122.

Serniclaes W, Van Heghe S, Mousty P, Carré R, SprengerCharolles L. (2004) Allophonic mode of speech perception in dyslexia. J Exp Child Psychol 87(4):336-361.

Tallal P. (1980) Auditory temporal perception, phonics, and reading disabilities in children. Brain Lang 9(2):182-198.

Tallal P. (1984) Temporal or phonetic processing deficit in dyslexia? That is the question. Appl Psycholinguist 5:167-169.

Tallal P. (2004) Improving language and literacy is a matter of time. Nat Rev Neurosci 5:721-728.

Tallal P, Gaab N. (2006) Dynamic auditory processing, musical experience and language development. Trends Neurosci 29(7): $382-390$

Vandermosten M, Boets B, Luts H, Poelmans H, Wouters J, Ghesquière P. (2011) Impairments in speech and nonspeech sound categorization in children with dyslexia are driven by temporal processing difficulties. Res Dev Disabil 32(2):593-603.

Wagner R, Torgesen J, Rashotte C. (2013) Comprehensive Test of Phonological Processing-Second Edition (CTOPP-2). London, UK: Pearson Clinical Assessment.

Weschler D. (2011) Wechsler Abbreviated Scale of IntelligenceSecond Edition (WASI-II). London, UK: Pearson Clinical Assessment.

White-Schwoch T, Woodruff Carr K, Thompson EC, Anderson S, Nicol T, Bradlow AR, Zecker SG, Kraus N. (2015) Auditory processing in noise: A preschool biomarker for literacy. PLoS Biol 13(7):1-17.

Ziegler JC, Goswami U. (2005) Reading acquisition, developmental dyslexia, and skilled reading across languages: a psycholinguistic grain size theory. Psychol Bull 131(1):3-29.

Ziegler JC, Pech-Georgel C, George F, Lorenzi C. (2009) Speech-perception-in-noise deficits in dyslexia. Dev Sci 12(5): 732-745. 